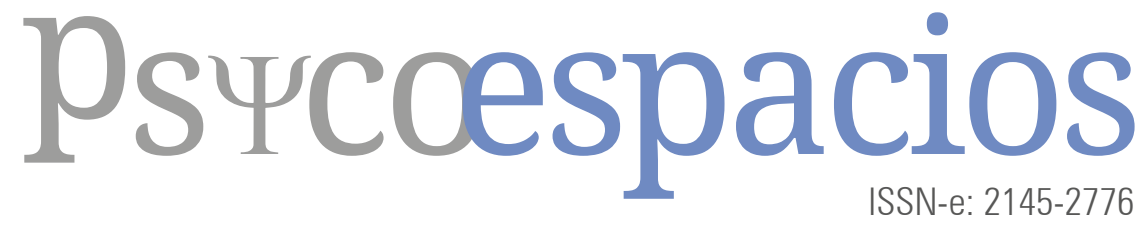

Vol. 15, № 26, enero-junio 2021 


\title{
Asociación entre inteligencia emocional, riesgo de depresión y condiciones sociodemográficas en estudiantes de psicología
}

\author{
Association between Emotional Intelligence, Depression Risk \\ and Sociodemographic Conditions in Psychology Students
}

\author{
Sandra Milena Castaño Ramírez ${ }^{\mathrm{a}}$ \\ Institución Universitaria de Envigado, Colombia \\ https://orcid.org/0000-0002-5466-2259 \\ Brian BaEna Múnera \\ Institución Universitaria de Envigado, Colombia \\ https://orcid.org/0000-0002-4924-2751
}

Recibido: 9 abril 2021 Aceptado: 20 agosto $2021 \bullet$ Publicado: 2 octubre 2021

\begin{abstract}
Cómo citar este artículo: Castaño Ramírez, S.M. y Baena Múnera, B. (2021). Asociación entre inteligencia emocional, riesgo de depresión y condiciones sociodemográficas en estudiantes de psicología. Psicoespacios, 15(26). https://doi.org/10.25057/21452776.1399
\end{abstract}

aAutor de correspondencia: smcastano@correo.iue.edu.co

\section{Resumen}

El propósito del artículo es determinar la asociación entre inteligencia emocional, riesgo de depresión y condiciones sociodemográficas en estudiantes de psicología. Se evalúa con la escala de inteligencia emocional percibida (TMMS-24) y el inventario de depresión de Beck II (BDI-II) a 307 estudiantes (245 mujeres y 62 hombres) de psicología de la Institución Universitaria de Envigado, los cuales cumplen la mayoría de edad. Los resultados obtenidos permiten identificar que los estudiantes con edades comprendidas entre los 18 y los 24 años presentan una poca claridad emocional en comparación con los participantes mayores de 35 años; dichos resultados sugieren que los jóvenes y los jóvenes adultos poseen un mayor riesgo de adquirir depresión que los de mayor edad. Además, se evidencia que, según el sexo, la regulación de las emociones podría variar. También se halla que existe menor riesgo de depresión para quienes tienen pareja sentimental en comparación con quienes no la tienen. Se encuentra que el riesgo a desarrollar depresión implica, entre muchas otras características, sentir y expresar las emociones percibidas adecuadamente. Los participantes de la muestra con riesgo de depresión prestan mucha atención a sus emociones y las expresan de una manera adecuada; sin embargo, tienen poca capacidad para comprenderlas y regularlas.

Palabras clave: Inteligencia emocional, depresión, atención emocional, reparación emocional, claridad emocional. 


\begin{abstract}
Objective: To determine how emotional intelligence, risk of depression and sociodemographic profiles are related in psychology students. Methodology: 307 legal age psychology students ( 245 women and 62 men) from Institución Universitaria de Envigado (municipality of Envigado, Colombia) were assessed using the Trait Meta-Mood Scale (TMMS-24) and the Beck Depression Inventory II (BDI-II). According with results, we could identify that students aged between 18 and 24 years (35.2\%) display little emotional clarity compared to participants older than 35 years. These results suggest that youths and young adults have a greater risk of acquiring depression in comparison with older age participants. Furthermore, we observed that emotion regulation ability may vary according to gender. Also, we found less risk of developing depression in students with legally established marital relationships compared to those not having a romantic partner. Risk of developing depression involves to feel and express a set of perceived emotions in a correct manner, among many other factors. Participants in the sample who are at risk for depression pay closer attention to their emotions and express them in a proper way, however they have little capacity to comprehend and regulate them.
\end{abstract}

Keywords: Emotional intelligence, depression, emotional attention, emotional repair, emotional clarity.

\section{Introducción}

La inteligencia emocional (IE) alude a la capacidad que tienen los seres humanos de reconocer tanto los propios sentimientos como los de otros; además, a la facultad de manejar adecuadamente las relaciones interpersonales y sociales. Es un término que integra habilidades adicionales a la inteligencia académica, que se define como una capacidad cognitiva medida por el cociente intelectual (Goleman, 1998).

El abordaje de la IE se puede agrupar en tres líneas de actuación. Principalmente se desarrollaron numerosos modelos teóricos que abordaban las bases fundamentales de la IE, que describían los elementos esenciales que una persona emocionalmente inteligente debía poseer. Luego de tener claras y argumentadas las bases teóricas definidas por los diferentes autores aparece la siguiente línea orientada a la producción de instrumentos de medición y evaluación de la IE de manera confiable. Gracias a los intereses personales de algunos investigadores poco a poco se comienzan a clasificar los métodos fiables y válidos para la medición de la IE dándole paso a la tercera línea de actuación, en la cual se exponen los estudios empíricos y posteriormente se explora la validez predictiva y la forma como se relaciona la IE con los diversos aspectos de la vida (Extremera Pacheco et al., 2004).

Salovey y Mayer (citados por Fernández Berrocal y Ramos Díaz, 1999) delimitan la inteligencia emocional como una parte de la inteligencia social, la que requiere de una capacidad para hacer control de las propias emociones, identificar las emociones de los demás y poder diferenciarlas; esto con el fin de usar la información que se obtenga para guiar los pensamientos y el comportamiento.

Según Mayer et al. (2001), las emociones contienen información acerca de las relaciones. En el momento en que existe un cambio en una relación con otra persona u objeto, también cambian las emociones. Esto sería independiente de si las relaciones son del pasado, del presente o a futuro (imaginadas). La información proveniente de estas relaciones puede ser usada, entonces, como base para razonar y resolver problemas que, posteriormente, se usarían para intensificar las actividades cognitivas.

Inicialmente Salovey y Mayer propusieron la existencia de la inteligencia emocional 
en el año 1990, sin recibir mucha atención del medio. Fue en 1995 cuando Goleman desarrolló este concepto traído por ellos y tuvo una gran recepción por parte de la comunidad y, desde ello, no ha cesado. Goleman ha adaptado su modelo a una versión más eficaz para demostrar su utilidad en el mundo laboral (Caruso et al., 2019).

En 1983 Salovey y Mayer plantean que la inteligencia se relaciona con 3 componentes: las características cognitivas internas, el rendimiento y las diferencias individuales, por lo cual, la definen como "las características cognitivas internas relativas a las diferencias individuales en el rendimiento, para la resolución de problemas" (Molero Moreno et al., 1998, p. 22). Estos dos autores introducen el término de inteligencia emocional, al explicar que esta es la capacidad de reconocer las señales y los acontecimientos emocionales propios y los de los otros; capacidad que, a través de un proceso de elaboración y producción emocional, genera un comportamiento adecuado y efectivo para las metas propias y del entorno (Salovey y Mayer, 1990).

De la misma forma, Salovey y Mayer propusieron inicialmente lo que se considerarían 4 dimensiones para describir la IE, siendo estos: percepción, comprensión, facilitación y regulación emocional (Extremera Pacheco y Fernández-Berrocal, 2013).

Percepción emocional: es la capacidad de percibir las emociones ajenas o propias, además de percibirlas provenientes de otros estímulos como la música, el arte, etc.

Facilitación emocional: tiene que ver con la habilidad para poder generar y sentir emociones que luego serán usadas en los procesos cognitivos que llevan a la toma de decisiones.

Comprensión emocional: trata de entender la información emocional, cómo se combinan e influyen entre sí las emociones, y apreciar los sentidos emocionales de las experiencias vividas.
Regulación emocional: hace referencia a la disposición que tienen los sujetos para experimentar estados emocionales positivos y negativos, para luego razonar sobre estos y determinar si la información que los acompañan es útil para sí mismo.

Actualmente la valoración de la IE se ha transformado en un aspecto crítico en la investigación sobre este tema. Se conocen diferencias individuales en las personas con respecto a sus competencias para poder atender a sus propios sentimientos y los de otras personas; además de comprenderlos de manera adecuada, modificar los estados de ánimo que provocan malestar y mantener por más tiempo los positivos. La importancia dada en los últimos años a los procesos internos como a la existencia de nuevos avances tecnológicos ha beneficiado el resurgimiento de la psicología de las emociones y su conexión con lo cognitivo. Durante décadas, en diversos ámbitos, se han tenido en cuenta las destrezas cognitivas, pues han adquirido un gran interés gracias a que la investigación sobre la inteligencia y la dicotomía entre lo emocional y lo racional ayudó a que el concepto de coeficiente intelectual (CI) se centrara en ellas (Fernández-Berrocal y Ramos Díaz, 1999).

La depresión es un trastorno psicológico que afecta en el mundo a 350 millones de personas; además, en Latinoamérica y el Caribe el $5 \%$ de la población adulta la padece y solo seis de cada diez son tratados. Según la Organización Mundial de la Salud (OMS), es la mayor causa de discapacidad en el mundo y cuando se presentan síntomas graves puede conducir al suicidio. Alrededor de 1 millón de personas se suicidan en el mundo al año. La adecuada atención a las personas con este trastorno tiene varias barreras, como la falta de capacitación y de servicios dedicados a la atención primaria y el estigma social que está ligado a los trastornos mentales (Organización Mundial de la Salud, 2012). Con respecto a la 
depresión y la edad, se evidenció que los pacientes jóvenes tienden a presentar depresión severa y moderada, la prevalencia de depresión en personas jóvenes se ha incrementado en los últimos años y es un trastorno que produce aislamiento social y puede, en casos muy severos, culminar en el suicidio (Acosta-Hernández et al., 2011). La depresión es un desorden que se presenta en alrededor de un 3 a $8 \%$ en personas jóvenes, mientras que en los adultos se presenta en aproximadamente un 4,5\% de la población (Ossa Hernandez et al., 2016).

También es importante saber que la OMS indica que la depresión tiene mayor prevalencia en mujeres $(5,1 \%)$ que en los hombres $(3,6 \%)$ y que es un trastorno mental de alta frecuencia cuyos síntomas son alteraciones en el estado de ánimo, declive de interés o disminución de placer, puede acompañarse de culpa o pérdida de amor propio, también se presentan problemas en el sueño, en el apetito, problemas en la capacidad de concentración y sensación de cansancio. Implica afectación en el desempeño laboral o académico y en la forma para enfrentar la vida. En estados graves se evidencia ideación suicida. Cuando los síntomas son graves requieren acompañamiento farmacológico y psicología clínica (Organización Mundial de la Salud, 2012).

Ahora bien, con respecto al DSM-5 (Asociación Psiquiátrica Americana, 2014), la depresión está ubicada en el apartado "trastornos depresivos", donde se pueden encontrar diversos tipos de trastornos: depresión mayor, distimia, trastorno disruptivo de regulación del ánimo, disforia premenstrual, trastornos depresivos inducidos por droga o medicación y trastornos depresivos debido a otras razones médicas.

Según el Ministerio de Salud y Protección Social (2015) en la Encuesta Nacional de Salud Mental, para el año 2015 en Colombia 25576 mujeres fueron atendidas por depresión moderada y 10753 hombres, com- parado al 2009 cuando se atendieron 13022 mujeres y 4071 hombres. En Antioquia, las cifras brindadas por el ministerio de salud en el año 2009 indican que las personas atendidas por depresión moderada eran de 7563 , a diferencia de otros departamentos principales como Valle del Cauca y en la capital donde sus cifras fueron de 964 y 2456 respectivamente. Para el 2015 esta cifra aumentó ampliamente a 15250 en Antioquia, 2560 en Valle del Cauca y 5199 personas atendidas en Bogotá. En esta se tamizó una población de 12 a 17 años, de 18 a 44 y de 45 años en adelante, detectando posibles casos de depresión y se encontró que la prevalencia de los síntomas fue mayor en los adolescentes (12 a 17 años) con un 15,8 \% (de 4 a 6 síntomas de depresión); seguido de un 3,2 \% de prevalencia en personas de 18 a 44 años (con más de 7 síntomas) y un 6,2\% en personas de 45 años o más (7 o más síntomas). En Medellín para el año 2018 se obtuvieron datos preliminares de las consultas externas a hospitales por distintos trastornos mentales. Entre estos datos se destaca que los casos referentes a trastornos del humor, o sea los afectivos, tuvieron una prevalencia del $35 \%$, lo que equivale exactamente a 57574 pacientes que consultaron por lo anteriormente mencionado. Para el mismo año se habían notificado de 2269 intentos de suicidio, presentándose una tasa anual de 90 casos por cada cien mil habitantes. Para añadir a este dato, se estima que ocurrieron alrededor de seis intentos de suicidio por día (Secretaría de Salud de Medellín, 2016).

Por otro lado, el Estudio Poblacional de Salud Mental Envigado de 2017 reconoce la depresión mayor como uno de los trastornos más frecuentes. Se ubica en el cuarto puesto entre las causas de discapacidad a nivel mundial y se calcula que ocupará el segundo puesto para el año 2020. Este trastorno tiene una relevante morbilidad y mortalidad. Se describe entonces que el suicidio es una de 
las causas de muerte en personas de 20 a 35 años, en las cuales el $50 \%$ se debe a la depresión. Dicho trastorno también es fundamental en la morbilidad y la mortalidad en cuanto a otras afecciones físicas como el infarto agudo de miocardio. Igualmente, se asocia con un consumo elevado de alcohol y sustancias psicoactivas, lo que lleva a dificultades en las relaciones interpersonales, familiares y laborales. La prevalencia de la depresión en el último año incluido en el estudio es de 2,2 $\%$ a $6,7 \%$. En la población general la prevalencia del trastorno es entre 10 y $20 \%$, es más común en mujeres: dos por cada hombre y se presenta más en adultos jóvenes (Torres de Galvis et al., 2018).

Según Beck, en los trastornos del estado de ánimo hay una distorsión en la forma como se procesa la información del medio. En las personas depresivas existe este sesgo ante sucesos que pueden implicar una pérdida o privación, por la que valoran negativamente, de forma excesiva, estas situaciones y las consideran globales, irreversibles y frecuentes. Se genera así una triada cognitiva negativa que está compuesta de una visión negativa de sí mismo, del mundo y del futuro (Beck et al., 2001).

En la actualidad, el espacio universitario, como el laboral o familiar, pone en el camino de los estudiantes una serie de obstáculos que les hacen requerir el uso de sus habilidades personales (emocionales, cognitivas, físicas). Estas características pueden desencadenar en esta población posibles caídas o colapsos que, sin la preparación personal adecuada, repercutirá en sus vidas no solamente en el ámbito académico sino también en su adaptación en general al medio. Por lo anterior, la inteligencia emocional sería de gran importancia, debido a que, según su nivel de desarrollo, los estudiantes resolverán de manera más acertada las situaciones de estrés, presión y frustración que soportan en la vida diaria.
Con relación a lo ya mencionado, las discusiones en investigaciones realizadas en los últimos años explican que los niveles de depresión bajos y el afrontamiento de situaciones de estrés implican un aumento en las habilidades de comprensión y manejo de las emociones. También concluyen que niveles de depresión altos implican una mayor tendencia a prestarle más atención a las emociones. Los resultados presentados en estas investigaciones aportan información relevante que visualiza la necesidad de fomentar la inteligencia emocional y sus habilidades, para con ello disminuir los síntomas anímicos negativos y con ello incrementar la percepción adecuada de manejo emocional (Barraza-López et al., 2017).

Teniendo en cuenta las anteriores consideraciones teóricas, el presente artículo busca determinar la asociación entre inteligencia emocional, riesgo de depresión y condiciones sociodemográficas en estudiantes de psicología.

\section{Metodología}

\section{Participantes}

Para la investigación se contó con una muestra de 307 estudiantes (245 mujeres y 62 hombres) de la Institución Universitaria de Envigado (Colombia).

La muestra se calculó con una probabilidad de éxito de $50 \%$, un nivel de confiabilidad de $95 \%$ y un margen de error de 5 $\%$. Para el estudio se tuvieron en cuenta los siguientes criterios de inclusión y exclusión, respectivamente:

Criterios de inclusión: a) estudiantes de psicología, b) aceptar voluntariamente la participación, c) estudiar en la Institución Universitaria de Envigado.

Criterios de exclusión: a) edad inferior a 18 años. 


\section{Procedimiento}

La investigación realizada es de enfoque cuantitativo, con un tipo de estudio descriptivo y un diseño transversal. Este proyecto fue liderado por el Semillero de Investigación de Neurociencias de la Institución Universitaria de Envigado. La participaron en el proyecto fue voluntaria por parte de cada estudiante que aseguró cumplir con los criterios de inclusión. La aplicación de las pruebas se realizó dentro de las aulas de clases, donde se les explico el fin de la investigación y se les proporciono el consentimiento informado, la encuesta sociodemográfica y los instrumentos BDI-II y el TMMS-24.

\section{Instrumentos}

Inventario de depresión de Beck II (BDI-II)

Es un autoinforme que solo requiere el uso de lápiz y papel; está compuesto por 21 ítems en escala Likert que evalúa síntomas de tristeza, sentimientos de fracaso, pérdida de placer, llanto, pensamientos de suicidio, culpa, entre otros. El inventario que fue propuesto por Beck ha sido uno de los instrumentos de más uso al momento de identificar y evaluar la severidad de la depresión. Este inventario se puede aplicar de forma individual o colectiva tanto de forma oral como escrita con lápiz y papel. Normalmente requiere entre 5 y 10 minutos para ser completado, pero dependiendo de la severidad de la depresión se podría tomar más tiempo. Una de las instrucciones es que el encuestado tenga en cuenta que las afirmaciones cubren el periodo de tiempo de las últimas dos semanas incluyendo el día en que se realiza el test. Cada pregunta tiene una escala de 4 puntos de 0 a 3 , siendo 0 la no identificación con el ítem y 3 la máxima identificación con este. La calificación mínima es 0 y la máxima es 63. El inventario posee unos puntajes de cor- te que permiten clasificar a los evaluados entre 4 grupos: 0-13, mínima depresión; 14-19, depresión leve; 20-28, depresión moderada; y 29-63, depresión severa. Para efectos de la investigación, al momento de realizar el análisis estadístico se tomó como "sin riesgo" los puntajes de 0 a 14 y a partir de 15 se contó el riesgo de desarrollar depresión (Melipillán Araneda et al., 2008).

Melipillán Araneda et al. (2008) hicieron un análisis de la consistencia interna del BDI-II; el resultado que obtuvieron en el coeficiente alfa de Cronbach correspondió a 0,91. Los autores replicaron este análisis para cada grupo participante por separado y observaron que valores en el coeficiente alfa de Cronbach fue de 0,91 en la muestra clínica, 0,89 en la muestra control y 0,89 en la muestra representativa. Para la validez se compararon los puntajes en la Escala BDIII de los participantes pertenecientes a las muestras clínica y control y observaron que la muestra clínica presenta un promedio de $29,01(\mathrm{DE}=13,49)$, mientras los de control un promedio de 19,18 $(\mathrm{DE}=11,58)$. Lo cual permite concluir que los participantes que tienen puntajes altos en el BDI-II son quienes tienen mayor probabilidad de dirigirse a atención en salud mental por presentar síntomas depresivos.

\section{La escala de inteligencia emocional percibida o Trait Meta-Mood Scale}

El TMms-24 está basado en el Trait Meta-Mood Scale de Salovey y Mayer; la escala original evalúa el metaconocimiento de las emociones mediante 48 ítems que miden la capacidad de regular las emociones, así como las destrezas que hacen que seamos conscientes de nuestras propias emociones. Su adaptación al español fue realizada por Fernández-Berrocal et al. (2004).

Esta escala está compuesta por 24 ítems de escala tipo Likert de cinco puntos (desde 1 
$=$ Nada de acuerdo, hasta $5=$ Totalmente de acuerdo), el test está compuesto por 3 dimensiones: atención emocional, que hace referencia a la percepción de las propias emociones, es decir, a la capacidad para sentir y expresar las emociones de forma adecuada; claridad de sentimientos, que alude a la percepción que se tiene sobre la comprensión de los propios estados emocionales; y reparación emocional como la capacidad percibida para regular los propios estados emocionales de forma correcta, con 8 ítems cada una de ellas (Fernández-Berrocal, Extremera y Ramos 2004).

Según el coeficiente de Cronbach la escala tMms-24 tiene una confiabilidad de 0,95 y sus subescalas se encuentran con una confiabilidad de 0,89 en comprensión, 0,86 en regulación y un 0,88 en percepción (Espinoza-Venegas et al., 2015).

\section{Plan de análisis}

Dentro de la investigación se realizó un análisis de datos por medio de técnicas de estadística descriptiva, ejecutando en primer lugar un análisis univariado para describir variables de la investigación como las condiciones sociodemográficas (edad, sexo, estado civil, semestre y estrato socioeconómico), las dimensiones de la inteligencia emocional (atención, claridad y reparación emocional, tratando los rangos poco, adecuado y excelente) y el riesgo de depresión, utilizando frecuencias y porcentajes. Luego de esto se llevó a cabo un modelo bivariado entre las variables sociodemográficas, las dimensiones de inteligencia emocional y depresión, además de asociaciones entre estas dos últimas, usando el estadístico Chi Cuadrado de Pearson, estableciéndose como significancia estadística un valor $\mathrm{p}$ menor a 0,05 . El software usado para hacer la base de datos y el posterior análisis estadístico fue el IBM SPSS v24.

\section{Resultados}

Dentro de los resultados sociodemográficos se puede observar (en la tabla 1) mayor participación de mujeres (254) en comparación con hombres (62), siendo un total de 307 estudiantes. En su mayoría se encuentran sin pareja (divorciado, soltero, viudo), mientras que una minoría tienen una relación legalmente constituida con un $12,7 \%$. Casi un cuarto de los estudiantes que participaron en el estudio vive en estrato socioeconómico medio. Los estudiantes que pertenecen a los semestres de primero a quinto son un poco más de la mitad y de sexto a décimo el resto. La mayoría de la muestra tiene entre 18 y 24 años, y una minoría son personas mayores de 35 años.

Tabla 1. Descripción de las condiciones sociodemográficas.

\begin{tabular}{lccc}
\hline & Variable & Frecuencia & Porcentaje \\
\hline \multirow{2}{*}{ Sexo } & Hombre & 62 & 20,2 \\
& Mujer & 245 & 79,8 \\
\hline Estado civil & Sin pareja & 268 & 87,3 \\
Con pareja & 39 & 12,7 & \\
\hline \multirow{2}{*}{ Estrato socioeconómico } & Bajo & 69 & 22,5 \\
& Medio & 232 & 75,6 \\
& Alto & 6 & 2 \\
Semestre & Entre primero y quinto & 179 & 58,3 \\
& Entre sexto y décimo & 128 & 41,7 \\
\multirow{2}{*}{ Edad } & Entre 18 y 24 años & 227 & 73,9 \\
& Entre 25 y 35 años & 67 & 21,8 \\
& Mayor a 35 años & 13 & 4,2 \\
\hline
\end{tabular}


En cuanto a dimensiones de inteligencia emocional, se observa (en la tabla 2) que más de la mitad de la muestra presentó adecuada atención emocional, adecuada claridad emocional y adecuada reparación emocional. Se encuentra una menor proporción de participantes con un nivel excelente en estas dimensiones.

Tabla 2. Descripción de las dimensiones de la inteligencia emocional.

\begin{tabular}{cccc}
\hline Dimensión & Atención emocional (AE) & Claridad emocional (CE) & Reparación emocional (RE) \\
\hline Poco & $79(25,7 \%)$ & $101(32,9 \%)$ & $69(22,5 \%)$ \\
Adecuado & $180(58,6 \%)$ & $163(53,1 \%)$ & $187(60,9 \%)$ \\
Excelente & $48(15,6 \%)$ & $43(14 \%)$ & $51(16,6 \%)$ \\
\hline
\end{tabular}

Luego de aplicar el BDI, se identificó (como se ve en la tabla 3) que la mayoría de la muestra estudiada no posee riesgo de desarrollar depresión, con un 68,1\% puntuado.

Tabla 3. Descripción de la variable de depresión.

\begin{tabular}{ccc}
\hline Depresión & Frecuencia & Porcentaje \\
\hline Sin riesgo & 209 & 68,1 \\
Con riesgo & 98 & 31,9 \\
\hline
\end{tabular}

Al realizar la asociación entre el sexo con las dimensiones de la inteligencia emocional y la depresión, se pudo hallar (como lo muestra la tabla 4) que no existen diferencias estadísticamente significativas entre hombres y mujeres en cuanto al riesgo de depresión, la atención emocional y la claridad emocional; sin embargo, se encuentra una asociación significativa entre el sexo y la dimensión de reparación emocional, identificándose que las mujeres presentan mayor probabilidad de tener una excelente reparación emocional en comparación con los hombres.

Tabla 4. Sexo y las variables de inteligencia emocional y depresión.

\begin{tabular}{|c|c|c|c|c|c|}
\hline Variable & & Hombres & Mujeres & Prueba de Chi cuadrado & Valor $\mathrm{P}^{*}$ \\
\hline \multirow{3}{*}{ Atención emocional } & Poco & $11(17,7 \%)$ & $68(27,8 \%)$ & \multirow{3}{*}{4,339} & \multirow{3}{*}{0,114} \\
\hline & Adecuado & $37(59,7 \%)$ & $143(58,4 \%)$ & & \\
\hline & Excelente & $14(22,6 \%)$ & $34(13,9 \%)$ & & \\
\hline \multirow{3}{*}{ Claridad emocional } & Poco & $21(33,9 \%)$ & $80(32,7 \%)$ & \multirow{3}{*}{1,227} & \multirow{3}{*}{0,542} \\
\hline & Adecuado & $35(56,5 \%)$ & $128(52,2 \%)$ & & \\
\hline & Excelente & $6(9,7 \%)$ & $37(15,1 \%)$ & & \\
\hline \multirow{3}{*}{ Reparación emocional } & Poco & $14(22,6 \%)$ & $55(22,4 \%)$ & \multirow{3}{*}{8,220} & \multirow{3}{*}{0,016} \\
\hline & Adecuado & $45(72,6 \%)$ & $142(58 \%)$ & & \\
\hline & Excelente & $3(4,8 \%)$ & $48(19,6 \%)$ & & \\
\hline \multirow{2}{*}{ Depresión } & Sin riesgo & $38(61,3 \%)$ & $171(69,8 \%)$ & \multirow{2}{*}{1,647} & \multirow{2}{*}{0,199} \\
\hline & Con riesgo & $24(38,7 \%)$ & $74(30,2 \%)$ & & \\
\hline
\end{tabular}

\footnotetext{
* Si valor $\mathrm{P}<0,05$ hay una asociación estadísticamente significativa entre las variables.
} 
Por otra parte, según los resultados obtenidos, no existe una asociación estadísticamente significativa entre la variable estado civil y las dimensiones de la IE, pero sí se encontró una asociación entre estado civil y riesgo de depresión: los estudiantes sin pareja legalmente constituida poseen mayor riesgo de depresión en comparación con aquellos que tienen pareja.

Tabla 5. Estado civil y las variables de inteligencia emocional y depresión.

\begin{tabular}{|c|c|c|c|c|c|}
\hline Variable & & Sin pareja & Con pareja & Prueba de Chi cuadrado & Valor $\mathrm{P}^{*}$ \\
\hline \multirow{3}{*}{ Atención emocional } & Poco & $69(25,7 \%)$ & $10(25,6 \%)$ & \multirow{3}{*}{1,055} & \multirow{3}{*}{0,590} \\
\hline & Adecuado & $155(57,8 \%)$ & $25(64,1 \%)$ & & \\
\hline & Excelente & $44(16,4 \%)$ & $4(10,3 \%)$ & & \\
\hline \multirow{3}{*}{ Claridad emocional } & Poco & $94(35,1 \%)$ & $7(17,9 \%)$ & \multirow{3}{*}{5,950} & \multirow{3}{*}{0,051} \\
\hline & Adecuado & $140(52,2 \%)$ & $23(59,0 \%)$ & & \\
\hline & Excelente & $34(12,7 \%)$ & $9(23,1 \%)$ & & \\
\hline \multirow{3}{*}{ Reparación emocional } & Poco & $65(24,3 \%)$ & $4(10,3 \%)$ & \multirow{3}{*}{5,235} & \multirow{3}{*}{0,073} \\
\hline & Adecuado & $162(60,4 \%)$ & $25(64,1 \%)$ & & \\
\hline & Excelente & $41(15,3 \%)$ & $10(25,6 \%)$ & & \\
\hline \multirow{2}{*}{ Depresión } & Sin riesgo & $175(65,3 \%)$ & $34(87,2 \%)$ & \multirow{2}{*}{7,501} & \multirow{2}{*}{0,006} \\
\hline & Con riesgo & $93(34,7 \%)$ & $5(12,8 \%)$ & & \\
\hline
\end{tabular}

"Si valor $\mathrm{P}<0,05$ hay una asociación estadísticamente significativa entre las variables.

Como se evidencia en la tabla 6, no se co; lo que indicaría que el estrato socioeconóencontraron asociaciones estadísticamente sig- mico de la vivienda no influye en la inteligencia nificativas entre las dimensiones de la IE, y la emocional y tampoco en el riesgo de sufrir una depresión con la variable estrato socioeconómi- depresión en la muestra de estudiantes.

Tabla 6. Estrato socioeconómico y las variables de inteligencia emocional y depresión.

\begin{tabular}{|c|c|c|c|c|c|c|}
\hline Variable & & Bajo & Medio & Alto & Prueba de Chi cuadrado & Valor $\mathbf{P}^{*}$ \\
\hline \multirow{3}{*}{ Atención emocional } & Poco & $15(21,7 \%)$ & $63(27,2 \%)$ & $1(16,7 \%)$ & \multirow{3}{*}{4,572} & \multirow{3}{*}{0,334} \\
\hline & Adecuado & $39(56,5 \%)$ & $138(59,5 \%)$ & $3(50,0 \%)$ & & \\
\hline & Excelente & $15(21,7 \%)$ & $31(13,4 \%)$ & $2(33,3 \%)$ & & \\
\hline \multirow{3}{*}{ Claridad emocional } & Poco & $27(39,1 \%)$ & $72(31,0 \%)$ & $2(33,3 \%)$ & \multirow{3}{*}{4,043} & \multirow{3}{*}{0,400} \\
\hline & Adecuado & $35(50,7 \%)$ & $126(54,3 \%)$ & $2(33,3 \%)$ & & \\
\hline & Excelente & $7(10,1 \%)$ & $34(14,7 \%)$ & $2(33,3 \%)$ & & \\
\hline \multirow{3}{*}{ Reparación emocional } & Poco & $18(26,1 \%)$ & $50(21,6 \%)$ & $1(16,7 \%)$ & \multirow{3}{*}{2,340} & \multirow{3}{*}{0,674} \\
\hline & Adecuado & $39(56,5 \%)$ & $143(61,6 \%)$ & $5(83,3 \%)$ & & \\
\hline & Excelente & $12(17,4 \%)$ & $39(16,8 \%)$ & $0(0,0 \%)$ & & \\
\hline \multirow{2}{*}{ Depresión } & Sin riesgo & $45(65,2 \%)$ & $159(68,5 \%)$ & $5(83,3 \%)$ & \multirow{2}{*}{0,925} & \multirow{2}{*}{0,630} \\
\hline & Con riesgo & $24(34,8 \%)$ & $73(31,5 \%)$ & $1(16,7 \%)$ & & \\
\hline
\end{tabular}

*Si valor $\mathrm{P}<0,05$ hay una asociación estadísticamente significativa entre las variables. 
Por el contrario, se encontró que las dimensiones de claridad emocional, reparación emocional y depresión sí guardan una asociación significativa con la edad (ver tabla 7). Se pudo observar que los jóvenes entre 18 y 24 años de edad tienen más probabilidad de pre- sentar poca claridad emocional y mayor riesgo de depresión, mientras que los estudiantes entre 25 y 35 años tienen más probabilidad de presentar poca reparación emocional en comparación con el grupo más joven y el de mayor edad.

Tabla 7. Edad y las variables de inteligencia emocional y depresión.

\begin{tabular}{|c|c|c|c|c|c|c|}
\hline Variable & & $\begin{array}{c}\text { Entre } 18 \text { y } 24 \\
\text { años }\end{array}$ & $\begin{array}{c}\text { Entre } 25 \text { y } \\
35 \text { años }\end{array}$ & $\begin{array}{c}\text { Mayor a } 35 \\
\text { años }\end{array}$ & $\begin{array}{l}\text { Prueba de Chi } \\
\text { cuadrado }\end{array}$ & Valor $\mathrm{P}^{*}$ \\
\hline \multirow{3}{*}{ Atención emocional } & Poco & $62(27,3 \%)$ & $14(20,9 \%)$ & $3(23,1 \%)$ & \multirow{3}{*}{$1,986^{\mathrm{a}}$} & \multirow{3}{*}{0,738} \\
\hline & Adecuado & $129(56,8 \%)$ & $42(62,7 \%)$ & $9(69,2 \%)$ & & \\
\hline & Excelente & $36(15,9 \%)$ & $11(16,4 \%)$ & $1(7,7 \%)$ & & \\
\hline \multirow{3}{*}{ Claridad emocional } & Poco & $80(35,2 \%)$ & $21(31,3 \%)$ & $0(0,0 \%)$ & \multirow{3}{*}{$14,967^{\mathrm{a}}$} & \multirow{3}{*}{0,005} \\
\hline & Adecuado & $118(52,0 \%)$ & $38(56,7 \%)$ & $7(53,8 \%)$ & & \\
\hline & Excelente & $29(12,8 \%)$ & $8(11,9 \%)$ & $6(46,2 \%)$ & & \\
\hline \multirow{3}{*}{ Reparación emocional } & Poco & $53(23,3 \%)$ & $16(23,9 \%)$ & $0(0,0 \%)$ & \multirow{3}{*}{$14,874^{\mathrm{a}}$} & \multirow{3}{*}{0,005} \\
\hline & Adecuado & $140(61,7 \%)$ & $41(61,2 \%)$ & $6(46,2 \%)$ & & \\
\hline & Excelente & $34(15,0 \%)$ & $10(14,9 \%)$ & $7(53,8 \%)$ & & \\
\hline \multirow{2}{*}{ Depresión } & Sin riesgo & $151(66,5 \%)$ & $45(67,2 \%)$ & $13(100,0 \%)$ & \multirow{2}{*}{6,375} & \multirow{2}{*}{0,041} \\
\hline & Con riesgo & $76(33,5 \%)$ & $22(32,8 \%)$ & $0(0,0 \%)$ & & \\
\hline
\end{tabular}

*Si valor $\mathrm{P}<0,05$ hay una asociación estadísticamente significativa entre las variables.

Por otra parte, como se evidencia en la indica que la inteligencia emocional y la detabla 8, no se encontró una asociación esta- presión se dan independientemente del nivel dísticamente significativa entre el semestre de estudio en la muestra.

y las dimensiones de IE y depresión, lo que

Tabla 8. Semestre y las variables de inteligencia emocional y depresión.

\begin{tabular}{|c|c|c|c|c|c|}
\hline Variable & & $\begin{array}{c}\text { Entre primero y } \\
\text { quinto }\end{array}$ & $\begin{array}{c}\text { Entre quinto y } \\
\text { décimo }\end{array}$ & $\begin{array}{c}\text { Prueba de Chi } \\
\text { cuadrado }\end{array}$ & Valor $\mathbf{P}^{*}$ \\
\hline \multirow{3}{*}{ Atención emocional } & Poco & $42(23,5 \%)$ & $37(28,9 \%)$ & \multirow{3}{*}{$4,045 a$} & \multirow{3}{*}{0,132} \\
\hline & Adecuado & $103(57,5 \%)$ & $77(60,2 \%)$ & & \\
\hline & Excelente & $34(19,0 \%)$ & $14(10,9 \%)$ & & \\
\hline \multirow{3}{*}{ Claridad emocional } & Poco & $62(34,6 \%)$ & $39(30,5 \%)$ & \multirow{3}{*}{$0,628 \mathrm{a}$} & \multirow{3}{*}{0,731} \\
\hline & Adecuado & $92(51,4 \%)$ & $71(55,5 \%)$ & & \\
\hline & Excelente & $25(14,0 \%)$ & $18(14,1 \%)$ & & \\
\hline \multirow{3}{*}{ Reparación emocional } & Poco & $36(20,1 \%)$ & $33(25,8 \%)$ & \multirow{3}{*}{$1,566 a$} & \multirow{3}{*}{0,457} \\
\hline & Adecuado & $111(62,0 \%)$ & $76(59,4 \%)$ & & \\
\hline & Excelente & $32(17,9 \%)$ & $19(14,8 \%)$ & & \\
\hline \multirow{2}{*}{ Depresión } & Sin riesgo & $114(63,7 \%)$ & $95(74,2 \%)$ & \multirow{2}{*}{$3,809 a$} & \multirow{2}{*}{0,051} \\
\hline & Con riesgo & $65(36,3 \%)$ & $33(25,8 \%)$ & & \\
\hline
\end{tabular}

*Si valor $\mathrm{P}<0,05$ hay una asociación estadísticamente significativa entre las variables. 
Finalmente, se encontró que el riesgo de depresión se asocia con la atención emocional, la claridad emocional y la reparación emocional (valor $\mathrm{p}<0,05$ ). Como se ve en la tabla 9, las personas con riesgo de depresión tienen más probabilidad de presentar una excelente atención emocional, pero poca claridad emocional y reparación emocional.

Tabla 9. Inteligencia emocional y depresión.

\begin{tabular}{|c|c|c|c|c|c|}
\hline Variable & & $\begin{array}{c}\text { Sin riesgo de } \\
\text { depresión }\end{array}$ & $\begin{array}{c}\text { Con riesgo de } \\
\text { depresión }\end{array}$ & $\begin{array}{c}\text { Prueba de Chi } \\
\text { cuadrado }\end{array}$ & Valor $\mathrm{P}^{*}$ \\
\hline \multirow{3}{*}{ Atención emocional } & Poco & $63(30,1 \%)$ & $16(16,3 \%)$ & \multirow{3}{*}{$10,661 \mathrm{a}$} & \multirow{3}{*}{0,005} \\
\hline & Adecuado & $121(57,9 \%)$ & $59(60,2 \%)$ & & \\
\hline & Excelente & $25(12,0 \%)$ & $23(23,5 \%)$ & & \\
\hline \multirow{3}{*}{ Claridad emocional } & Poco & $49(23,4 \%)$ & $52(53,1 \%)$ & \multirow{3}{*}{$26,855 a$} & \multirow{3}{*}{0,000} \\
\hline & Adecuado & $125(59,8 \%)$ & $38(38,8 \%)$ & & \\
\hline & Excelente & $35(16,7 \%)$ & $8(8,2 \%)$ & & \\
\hline \multirow{3}{*}{ Reparación emocional } & Poco & $33(15,8 \%)$ & $36(36,7 \%)$ & \multirow{3}{*}{$18,907 a$} & \multirow{3}{*}{0,000} \\
\hline & Adecuado & $134(64,1 \%)$ & $53(54,1 \%)$ & & \\
\hline & Excelente & $42(20,1 \%)$ & $9(9,2 \%)$ & & \\
\hline
\end{tabular}

*Si valor $\mathrm{P}<0,05$ hay una asociación estadísticamente significativa entre las variables.

\section{Discusión}

En su investigación en estudiantes de Psicología, Aguilar-Luzón et al. (2012) encontraron diferencias significativas respecto a la edad. Hallaron que a mayor edad existe mayor claridad y regulación emocional; en el presente estudio se encontró que lo más jóvenes tenían más poca atención emocional y mayor riesgo de depresión.

García Cruz et al. (2017) encontraron que el sexo femenino tiene mayor vulnerabilidad ante la posibilidad de desarrollar depresión; asimismo, lo expusieron Gómez-Romero et al. (2018), quienes reportaron que las mujeres presentan más sintomatología depresiva que los hombres. Es importante mencionar que, en la presente investigación y respecto al sexo, las mujeres puntuaron mayor reparación emocional que los hombres. Ante esto, Goleman (1998) menciona que las mujeres que son emocionalmente inteligentes tienen una visión positiva de ellas mismas y poseen un sentido claro de la vida. Al expresar sus sentimientos de forma adecuada y soportar bien la tensión, tendrían un equilibrio social. Son lo suficientemente seguras de sí mismas para mostrarse alegres, espontáneas y abiertas a nuevas experiencias.

Por transmisión cultural y familiar se les ha considerado a las mujeres como más emocionales. Esta afirmación se justifica en el hecho de que están en un mayor contacto con sus sentimientos, permitiéndose ser más expresivas y reconocer adecuadamente las emociones en los demás (Candela Agulló et al., 2002).

Sin embargo, los resultados de los autoinformes realizados a distintos grupos poblacionales y culturas dejan ver una diferencia en los resultados que no permite generalizar una premisa acerca de estas dos variables. En algunos de estos estudios, el sexo femenino se muestra con una excelente habilidad para dirigir y manejar las emociones propias y las del resto. Así mismo, existen otros en los que ocurre lo 
opuesto, y este sexo muestra mayor capacidad en la atención emocional y percepción de la emocionalidad externa. Ante esta variedad de resultados, Sánchez Núñez et al. (2017) sugieren que se puede deber a las diferencias sociodemográficas y culturales en la muestra y también al instrumento que se use.

Siguiendo las ideas previamente mencionadas, Barraza-López et al. (2017), en su estudio sobre la relación entre IE y depresión-ansiedad y estrés, encontraron que, así como en la presente investigación, a menores síntomas de depresión mayor control y manejo de las emociones; también a mayor riesgo de depresión hay más atención emocional.

Rodríguez de Alba y Suárez Colorado (2012) se encargaron de investigar la IE y el riesgo de depresión también en estudiantes universitarios. Concluyeron que mientras más puntuaciones altas en claridad y reparación emocional, menor nivel de depresión es evidenciado en los participantes.

Batool y Khalid (2009) y Mamani-Benito et al. (2018) concluyeron en sus investigaciones que la inteligencia emocional sirve como predictor de la depresión. Esta conclusión se puede relacionar con el presente trabajo, teniendo en cuenta la asociación significativa que existe entre las tres dimensiones de la IE y la depresión. Las investigaciones mencionadas dejan claros los beneficios de la IE en términos de salud mental y ajuste psicosocial. Las personas que son inteligentes emocionalmente, al saber gestionar mejor sus inconvenientes emocionales, informan menos síntomas correspondientes a ansiedad, depresión, ideación suicida, entre otros.

Los bajos resultados en las habilidades emocionales podrían suponer una baja capacidad adaptativa en la vida cotidiana y más posibilidades de desarrollar conductas de riesgo. Se encontraron relaciones significativas entre depresión e IE, correlacionándose principalmente la dimensión de atención emocional con el riesgo a depresión. Esto puede aumentar la conducta rumiativa, la cual se relaciona con pensamientos constantes sobre lo que se está sintiendo emocionalmente, pero sin pensar en ningún método efectivo para afrontarlo; el presentar pensamientos rumiativos como reacción a un estado emocional de tristeza, empeora y permite que se prolonguen los síntomas que acompañan a este estado. Esta respuesta trata de prestar atención de forma continua y recurrente a los sentimientos, además de las causas y consecuencias del estado de ánimo en el que se encuentre; esto sin tener especial cuidado por aliviarlos o resolver el problema de raíz. Este tipo de estilo tendría mayor vulnerabilidad a experimentar sintomatología ansiosa y depresiva (Extremera et al., 2006).

Por ello y luego de encontrarse una asociación significativa entre la variable de IE y el riesgo a depresión, se hace de suma importancia que, en la formación de los individuos desde su fase inicial en el crecimiento, se les brinde los recursos para lograr un aprendizaje que permita el desarrollo de competencias esenciales para su bienestar. La adaptación social y el permitirse crear asertivas soluciones a los conflictos interpersonales serían el resultado de un adecuado aprendizaje de las habilidades emocionales planteadas inicialmente.

\section{Conclusiones}

Los resultados de la investigación permitieron identificar que la IE es un factor clave para prevenir y mitigar el riesgo de desarrollar depresión. De esta manera, las tres dimensiones de la IE se asocian significativamente con la posibilidad de desarrolla o no la depresión. Se pudo corroborar que la atención emocional es una dimensión que los participantes con riesgo de depresión puntúan alta; por lo tanto, las personas que sienten y expresan las emociones y les ponen mucha atención y las rumian y hablan sobre ellas, tienen más ries- 
go de tener depresión. De la misma manera, aquellas personas con alto riesgo de depresión poseen baja comprensión de las emociones que están sintiendo y se les presenta dificultad para manejarlas o regularlas. Las altas puntuaciones en atención emocional en las personas con riesgo de depresión se relacionaron con una baja habilidad para saber qué hacer con esas emociones.

Sería de gran aporte el desarrollo de programas de prevención e intervención sobre depresión, autoestima, entrenamiento en habilidades sociales y manejo de emociones, con el fin de fomentar un nivel socioemocional adecuado en los estudiantes, y de esta manera prevenir la aparición de problemas psicológicos y emocionales.

Igualmente, es importante realizar más estudios sobre la relación de la IE con otras variables psicológicas y educativas con el fin de dimensionar de una mejor manera causas originadas en otros factores psicosociales que afecten directa o indirectamente el riesgo a tener depresión. Como bien se estableció, esta es multicausal y a pesar de que la IE funcione como predictora de la depresión, no es la única variable para tener en consideración en cuanto a su desarrollo.

Futuras investigaciones longitudinales con la intención de aplicar estrategias que permitan el desarrollo de las habilidades emocionales serían valiosas para prevenir psicopatologías y mejorar la elaboración de las estrategias de afrontamiento y percepción del mundo y de sí mismo.

Finalmente, es recomendable replicar esta investigación en las diferentes facultades de la Institución Universitaria de Envigado y en las diferentes universidades de la ciudad y del país con el fin de analizar las diferencias o similitudes entre las carreras investigadas y cómo, en función de dicho contenido académico, se ven afectadas la inteligencia emocional y definir si existe o no riesgo de depresión.

\section{Referencias}

Acosta-Hernández, M. E., Mancilla-Percino, T., Correa-Basurto, J., Saavedra-Vélez, M., Ramos-Morales, F. R., Cruz-Sánchez, J. S. y Duran-Niconoff, S. (2011). Depresión en la infancia y adolescencia: enfermedad de nuestro tiempo. Archivos de Neurociencias, 16(3), 156-161. https://www. medigraphic.com/cgi-bin/new/resumen. cgi? IDARTICULO $=32548$

Aguilar-Luzón, M., Calvo-Salguero, A. y Monteoliva-Sánchez, A. (2012). La inteligencia emocional percibida y su relación con el apego adulto. Psicología Conductual, 20(1), 119-135. https://www. researchgate.net/profile/Maria_Carmen_ Aguilar-Luzon2/publication/267515020 La_inteligencia_emocional_percibida_y_ su_relacion_con_el_apego_adulto/ links/560d091708aece4e70e06639/ La-inteligencia-emocional-percibida-y-surelacion-con-el-apego-adulto.pdf

Asociación Psiquiátrica Americana. (2014). DSM-5. Manual diagnóstico y estadístico de los trastornos mentales. Editorial Médica Panamericana.

Barraza-López, R. J., Muñoz-Navarro, N. A. y Behrens-Pérez, C. C. (2017). Relación entre Inteligencia Emocional y depresión-ansiedad y estrés en estudiantes de medicina de primer año. Revista chilena de neuro-psiquiatría, 55(1), 18-25. https://scielo.conicyt.cl/ scielo.php? script $=$ sci_abstract $\&$ pid $=$ S0717 92272017000100003\&lng $=$ es\&nrm $=$ iso

Batool, S. S. y Khalid, R. (2009). Low emotional intelligence: A risk factor for depression. Journal of Pakistan Psychiatric Society, 6(2), 65-72. https://www.researchgate.net/ publication/306189786_Low_emotional_ intelligence_A_risk_factor_for_depression

Beck, A. Rush, J. Shaw, B. y Emery, G. (2001). Terapia Cognitiva de la depresión. Descleé de Brouwer.

Candela Agulló, C., Barberá Heredia, E., Ramos López, A. y Sarrió Catalá, M. (2002). Inteligencia emocional y la variable género. Revista Electrónica de Motivación y Emoción. 
5(10). http://reme.uji.es/articulos/ acandc2272105102/texto.html

Caruso, D. R., Mayer, J. D., Bryan, V., Phillips, K. G. y Salovey, P. (2019). Measuring emotional and personal intelligence. En M. W. Gallagher y S. J. Lopez (Eds.), Positive psychological assessment: A handbook of models and measures (pp. 233-245). https:// doi.org/10.1037/0000138-015

Espinoza-Venegas, M., Sanhueza-Alvarado, O., Ramírez-Elizondo, N. y Sáez-Carrillo, K. (2015). Validación de constructo y confiabilidad de la escala de inteligencia emocional en estudiantes de enfermería. Revista Latino-Americana de Enfermagem, 23(1), 139-147. https://www.scielo.br/pdf/ rlae/v23n1/es_0104-1169-rlae-23-01-00139. pdf

Extremera, N., Fernández-Berrocal, P., RuizAranda, D. y Cabello, R. (2006). Inteligencia emocional, estilos de respuesta y depresión. Ansiedad y Estrés, 12(2-3), 191-205. https:// emotional.intelligence.uma.es/documentos/ PDF26estilos_respuesta_depresion.pdf

Extremera Pacheco, N., Fernández-Berrocal, P., Mestre Navas, J. M. y Guil Bozal. R. (2004). Medidas de evaluación de la Inteligencia Emocional. Revista Latinoamericana de Psicología, 36(2), 209-228. https://www. redalyc.org/pdf/805/80536203.pdf

Extremera Pacheco, N. y Fernández-Berrocal, P. (2013). Inteligencia Emocional en adolescentes. Revista Padres y Maestros, 352, 34-39. https://revistas.comillas. edu/index.php/padresymaestros/article/ view/1170/993

Fernández-Berrocal, P., Extremera, N. y Ramos, N. (2004). Validity and Reliability of the Spanish Modified Version of the Trait Meta-Mood Scale. Psychological Reports, 94(3), 751-755. https://doi.org/10.2466/pr0.94.3.751-755

Fernández Berrocal, P. y Ramos Díaz, N. (1999). Investigaciones empíricas en el ámbito de la Inteligencia Emocional. Ansiedad y Estrés, 5(23), 247-260. https://emotional.intelligence. uma.es/documentos/PDF16Investigaciones empiricas.pdf
García Cruz, R., Valencia Ortiz, A. I., HernándezMartínez, A. y Rocha Sánchez, T. E. (2017). Pensamiento rumiativo y depresión entre estudiantes universitarios: representando el impacto del género. Revista Interamericana de Psicología, 51(3), 406-416. https://www. redalyc.org/pdf/284/28455448011.pdf

Goleman, D. (1998). La práctica de la Inteligencia Emocional. Kairós.

Gómez-Romero, M. J., Limonero, J. T., ToroTrallero, J., Montes-Hidalgo, J. y TomásSábado, J. (2018). Relación entre Inteligencia Emocional, afecto negativo y riesgo suicida en jóvenes universitarios. Ansiedad y Estrés, 24(1), 18-23. https://www.researchgate. net/publication/321216231_Relacion_entre inteligencia_emocional_afecto_negativo_y_ riesgo_suicida_en_jovenes_universitarios Relationship_between_emotional_ intelligence_negative_affect_and_suicidal_ risk_in_university_students

Mamani-Benito, O. J., Brousett-Minaya, M. A., Ccori-Zúñiga, D. N. y Villasante-Idme, K. S. (2018). La Inteligencia Emocional como factor protector en adolescentes con ideación suicida. Duazary, 15(1), 39. http://revistas. unimagdalena.edu.co/index.php/duazary/ article/view/2142

Mayer, J. D., Salovey, P., Caruso, D. R. y Sitarenios, G. (2001). Emotional intelligence as a standard intelligence. Emotion, 1(3), 232-242. https://doi.org/10.1037/1528-3542.1.3.232

Melipillán Araneda. R., Cova Solar. F., Rincón González, P. y Valdivia Peralta, M. (2008). Propiedades Psicométricas del Inventario de depresión de Beck-II en Adolescentes Chilenos. Terapia psicológica, 26(1), 5969. https://dx.doi.org/10.4067/S071848082008000100005

Ministerio de Salud y Protección Social de Colombia. (2015). Encuesta Nacional de Salud Mental 2015. MinSalud, Colciencias y Universidad Javeriana. https://www.minsalud.gov.co/ sites/rid/Lists/BibliotecaDigital/RIDE/DE/ encuesta-nacional-salud-mental-ensm-2015. zip

Molero Moreno, C., Saiz Vicente, E. y Esteban Martínez, C. (1998). Revisión histórica del 
concepto de inteligencia: una aproximación a la Inteligencia Emocional. Revista latinoamericana de psicología, 30(1), 1130. https://dialnet.unirioja.es/servlet/ articulo? codigo $=2834736$

Organización Mundial de la Salud. (2012, 9 de octubre). Día Mundial de la Salud Mental: la depresión es el trastorno mental más frecuente. https://www.paho.org/hq/ index.php?option $=$ com_content\&view $=\mathrm{a}$ rticle\&id = 7305:2012-dia-mundial-saludmental-depresion-trastorno-mental-masfrecuente\&Itemid $=1926 \&$ lang $=\mathrm{es}$

Ossa Hernandez, A. M., Sanchez Ochoa, A., Mejia Vasco, C. E. y Bareño Silva, J. (2016). Prevalencia de depresión y sus factores asociados en adolescentes entre 13 y 17 años en Colombia año 2003. CES Salud Pública, 7(1), 37-48. https://doi.org/10.21615/3282

Rodríguez de Alba, U. y Suárez Colorado, Y. (2012). Relación entre Inteligencia Emocional depresión y rendimiento académico en estudiantes de psicología. Psicogente, 15(28), 348-359. https://www.redalyc.org/ pdf/4975/497552361011.pdf

Salovey, P. y Mayer, J. D. (1990). Emotional intelligence. Imagination, Cognition and
Personality, 9(3), 185-211. https://doi. org/10.2190/DUGG-P24E-52WK-6CDG

Sánchez Núñez, M. T., Fernández-Berrocal, P., Montañés Rodríguez, J. y Latorre Postigo, J. M. (2017). ¿Es la inteligencia emocional una cuestión de género? Socialización de las competencias emocionales en hombres y mujeres y sus implicaciones. Electronic Journal of Research in Education Psychology, 6(15). https://doi.org/10.25115/ejrep. v6i15.1287

Secretaría de Salud de Medellín. (2016). Plan Territorial de Salud 2016-2019. Revista Salud Pública de Medellín, 6(1), 8-203. https://www.medellin.gov.co/irj/go/km/ docs/pccdesign/SubportaldelCiudadano_2/ P 1 a n d e D e s a r r o 11 o 0 _ $19 /$ ProgramasyProyectos/Shared\%20Content/ Plan\%20de\%20Salud\%202016/2016-2019/ Plan\%20Territorial\%20de\%20Salud $\% 20$ 2016\%20-\%202019.pdf

Torres de Galvis, Y., Restrepo Bernal, D., Castaño Pérez, G., Sierra Hincapié, G. M., Buitagro Salazar, C., Salas Zapata, C. y Bareño Silva, J. (2018). Estudio Poblacional de Salud Mental Envigado 2017. Editorial CES. https:// repository.ces.edu.co/handle/10946/3788 